\title{
Magnetic confinement of the solar tachocline: influence of turbulent convective motions
}

\author{
Antoine Strugarek ${ }^{1}$, Allan Sacha Brun ${ }^{1}$ and Jean-Paul Zahn ${ }^{2,1}$ \\ ${ }^{1}$ Laboratoire AIM Paris-Saclay, CEA/Irfu Université Paris-Diderot CNRS/INSU, \\ F-91191 Gif-sur-Yvette (email: antoine.strugarek@cea.fr) \\ ${ }^{2}$ LUTh, Observatoire de Paris-Meudon, France
}

\begin{abstract}
We present the results of 3D simulations, performed with the ASH code, of the nonlinear, magnetic coupling between the convective and radiative zones in the Sun, through the tachocline. Contrary to the predictions of Gough \& McIntyre (1998), a fossil magnetic field, deeply buried initially in the solar interior, will penetrate into the convection zone. According to Ferraro's law of iso-rotation, the differential rotation of the convective zone will thus expand into the radiation zone, along the field lines of the poloidal field.
\end{abstract}

Keywords. Sun: magnetic fields - stars: evolution - stars : rotation

\section{Two scenarios for the tachocline confinement}

Spiegel \& Zahn (1992) were the first to develop the concept of the tachocline, the thin region that links in the Sun the differential rotation of the convection zone with the quasi uniform rotation of the radiation zone. They showed that, due to thermal diffusion, this differential rotation would spread far into the deep interior. Since this is not observed, Spiegel \& Zahn (1992) invoked an anisotropic turbulence that would erode the latitudinal gradient of angular velocity. Gough \& McIntyre (1998) questioned this anisotropic turbulent momentum transport, quoting examples from geophysical studies to rebut this scenario. They proposed that the confinement of the tachocline is achieved by a fossil magnetic field buried in the radiation zone. However, numerical simulations carried out by Garaud (2002) and Brun \& Zahn (2006) did not confirm this magnetic scenario. Revisiting GM98, it appeared that the meridional circulation of the convection zone was a key factor in confining the magnetic field inside the radiation zone, and that the simulations should allow for radial flow between these two zones. As a matter of fact, when implementing an ad-hoc meridional flow in the convection zone, Sule et al. (2005) and Garaud \& Garaud (2008) managed to numerically recover the GM98 results. To circumvent these boundary conditions issues, we present here the first 3D MHD numerical simulations that couple the solar radiation zone with the convection zone, both regions being treated in the most realistic way. These simulations were performed with the ASH code (for Anelastic Spherical Harmonics, Brun et al. (2004)).

\section{Evolution of a magnetic field of fossil origin}

We start the simulation with a relaxed convection zone, and with a dipolar magnetic field confined in the radiation zone, in equipartition of energy with the rotation in that zone ( see figure 1(a). As the model evolves, we observe the development in the tachocline of a strong toroidal magnetic field, which is due to shearing of the poloidal field by the differential rotation; it is shown in figure 1(c). In that figure the instantaneous meridional circulation (i.e. the azimuthally averaged meridional velocity) is drawn in black. There 
is no sign of polar confinement of the magnetic field as predicted by Wood \& McIntyre (2010). The amplitude of the meridional circulation, near the poles, varies from $18 \mathrm{~m} . \mathrm{s}^{-1}$ at the base of the convective zone to $0.06 \mathrm{~m} . \mathrm{s}^{-1}$ at the base of the tachocline, thus losing 3 orders of magnitude in 0.03 solar radius. Although our tachocline is somewhat too thick, diffusivity values have been selected to approach as much as possible the solar parameters in such a global 3D simulation.

As the magnetic field evolves, it is at the same time advected, sheared and diffused through the tachocline into the convective zone (Fig. 1(d)). Magnetic pumping certainly exists in the equatorial region, but is not efficient enough to confine the magnetic field there. As seen in figure 1(b), magnetic field lines open into the convective zone. According to Ferraro's law, the differential rotation of the convective zone spreads into the radiative zone through the Maxwell tensor, and it achieves that much faster than if it were transported solely by thermal diffusion.

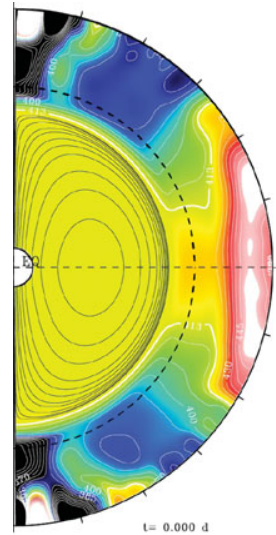

(a)

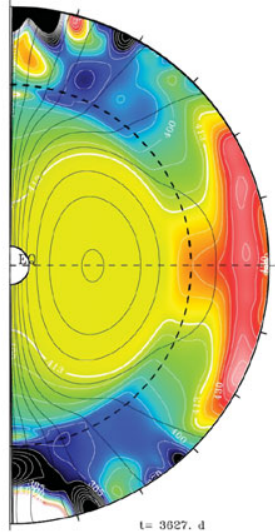

(b)

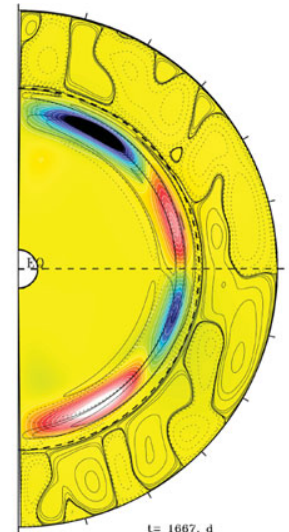

(c)

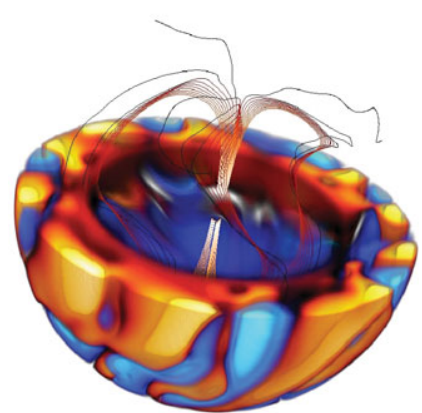

(d)

Figure 1. Meridional snapshots of azimuthal averages of angular velocity (colored background) and magnetic field lines (black); (a) initial field configuration, (b) after around 180 convective turnover times. Figure (c) shows the magnetopause (GM98) at the same epoch, with the instantaneous meridional circulation in black lines. Figure (d) displays a snapshot of magnetic field lines, and of the azimuthal velocity field $v_{\varphi}$ in colored volume; it illustrates the three-dimensional character of the penetration into the convection zone of the magnetic field.

Our simulations demonstrate that the velocity field in the convection zone cannot be reduced to a steady meridional flow, when the scope is to describe the penetration into that zone of an interior field of fossil origin. Instead of being deflected by this laminar flow, the field pervades the whole convection zone, and it imprints the differential rotation on the radiation zone. Since this is not observed, we conclude that the magnetic scenario must be refined. More detailed results are to appear soon (Strugarek et al. (2010)).

We acknowledge funding by ERC through grant STARS2 207430.

\section{References}

Brun, A. S., Miesch, M. S., \& Toomre, J. 2004, ApJ Series, 614, 1073

Brun, A. S. \& Zahn, J.-P. 2006, A\&A, 457, 665

Garaud, P. 2002, MNRAS, 329, 1

Garaud, P. \& Garaud, J.-D. 2008, MNRAS, 391, 1239

Gough, D. O. \& McIntyre, M. E. 1998, Nature, 394, 755
Miesch, M. S. 2003, ApJ, 586, 663

Spiegel, E. A. \& Zahn, J.-P. 1992, A\&A, 265, 106

Strugarek, A., Brun, A. S., \& Zahn, J. P. 2010, submitted to A\&A

Sule, A., Rudiger, G. \& Arlt, R. 2005, A\&A, 437, 1061

Wood, T. \& McIntyre, M. 2010, submitted to NJP 